\title{
Primum non nocere: Tacto rectal en niños ¿Es realmente necesario?*
}

\author{
Drs. STEPHANIE SUBERCASEAUX V. ${ }^{1}$, SERGIO ZÚÑIGA R. ${ }^{1}$ Int. M. CECILIA RIUTORT K. ${ }^{2}$
}

1 Sección Cirugía Pediátrica Hospital Clínico Universidad Católica de Chile.

2 Interna de $7^{\circ}$ año de la Escuela de Medicina Universidad Católica de Chile. Santiago, Chile.

\begin{abstract}
\section{Primum non nocere: Digital rectal examination in children. Is it really necessary?}

Traditionally we have performed digital rectal examination to children in a great number of conditions. We review the evidence supporting this practice like in acute abdomen, constipation, gastrointestinal bleeding, irritability, trauma, among others. However there is not enough evidence to support the rutinary digital rectal examination and is probably not always necessary.
\end{abstract}

Key words: Digital rectal examination, children.

\section{Resumen}

Tradicionalmente se ha indicado practicar el tacto rectal en niños en una amplia variedad de afecciones. Se realizó una revisión de la evidencia existente en la literatura respecto a este examen en diferentes entidades infantiles, como es el caso del abdomen agudo, la constipación, la hemorragia gastrointestinal, la irritabilidad y el trauma, entre otros. La evidencia respecto a su real utilidad es escasa y tal vez, sea probable, que la práctica rutinaria del examen rectal no sea necesaria.

Palabras clave: Tacto rectal, niños.

\section{Introducción}

Específicamente, el tacto rectal es definido como la exploración del recto de un paciente con el dedo del examinador ${ }^{1}$. En ediciones de textos clásicos de cirugía, es posible encontrar frases como "muchas veces, la omisión del examen rectal ha sido causa de arrepentimiento" o "el examen rectal es una parte esencial del examen fisico de todo paciente con sospecha de apendicitis"2. También hemos leído "el examen rectal continúa siendo parte esencial de un examen fisico completo"3. Y aún en libros de pediatría se citaba la exploración rectal como un elemento importante y que "nunca debe faltar en el examen de un escolar con abdomen agudo".

La información aportada por un examen rectal en niños en distintas circunstancias y sobre todo a distintas edades, al parecer, no es del todo tan evidente. Los niños pueden reaccionar de forma negativa frente a distintas intervenciones médicas, especialmente si ya han tenido experiencias previas negativas $^{5}$. El tacto rectal puede resultar en una

*Recibido el 2 de Noviembre de 2009 y aceptado para publicación el 30 de Noviembre de 2009.

Correspondencia: Dr. Sergio Zúñiga R.

Marcoleta 352 interior, 2do piso, Santiago, Chile.

E-mail: zuniga@med.puc.cl 
situación significativamente estresante tanto para el niño como para los padres que lo presencian ${ }^{6}$. Más aún, estudios correlacionan el dolor experimentado durante los procedimientos médicos en la niñez, con miedo a estos en la etapa adulta ${ }^{7}$.

El objetivo de la siguiente revisión bibliográfica, es analizar y actualizar algunas de las distintas indicaciones que existen para realizar un tacto rectal en los niños y evaluar una potencial utilidad de este.

\section{Abdomen agudo}

El gran clínico norteamericano William Osler, en su libro Principios y prácticas de la medicina (1901), establece que el examen rectal debe realizarse en toda instancia, al referirse a la apendicitis ${ }^{8}$. $\mathrm{La}$ principal labor del consultor es realizar el examen rectal que usted no hizo", es otra frase que utiliza Osler al referirse al tacto rectal ${ }^{9}$. Sin embargo, en el mismo texto este autor menciona que la "examination per rectum" rara vez da información de valor en las primeras etapas de una apendicitis ${ }^{8}$.

En las últimas décadas se ha cuestionado el valor del examen rectal en niños que se presentan con un cuadro clínico sugerente de abdomen agudo ${ }^{10}$. Dickson y Mackinlay en una serie de pequeños con apendicitis aguda, encontraron que más del $90 \%$ de los casos fueron diagnosticados por la historia clínica y el examen abdominal. En esta misma serie, en sólo $12 \%$ de los pacientes el examen rectal estuvo alterado. Los autores además describen que el tacto rectal provocó gran incomodidad, definida como llantos y gritos en $1 / 3$ de los niños y concluyen que el examen debería ser omitido de la evaluación de rutina de un paciente con abdomen agudo y que sólo estaría justificado en cuadros clínicos poco claros de apendicitis aguda. Además, afirman que el examen rectal es un mal examen como indicador precoz de sepsis pelviana tras la apendicectomía ${ }^{11}$. Bonello y Abrams publicaron en 1979, los resultados del tacto rectal en 495 pacientes con sospecha de apendicitis. En esta serie, el tacto rectal estuvo alterado en $46 \%$ de los pacientes con apendicitis aguda y en 53\% de aquellos con apéndices normales, de manera que el examen no confirma ni descarta el diagnóstico de apendicitis aguda ${ }^{12}$. Últimamente se ha visto que el manejo no se modifica por el resultado del tacto rectal y que lo que más se asocia a la apendicitis es la rigidez de la pared abdominal ${ }^{13}$. De esta forma, el examen rectal debiera ser considerado como parte de una "investigación" más que parte del examen físico de rutina y debiera ser sólo realizado cuando su resultado pueda cambiar el manejo del paciente ${ }^{14}$. Además, la interpreta- ción de la sensibilidad en el tacto rectal pediátrico puede ser difícil dada la incomodidad relacionada con el propio examen y la falta de colaboración de los pequeños. Actualmente, la observación del niño, en el tiempo, y en lo posible hospitalizado, constituye una de las herramientas más útiles en pacientes con sospecha de abdomen agudo. Con ello se reduce el número de laparotomías negativas y se evita la necesidad del examen rectal de rutina ${ }^{15}$.

\section{Constipación}

Es un problema clínico común y explica hasta el $30 \%$ de las causas de derivación a gastroenterología infantil ${ }^{16}$. La mayoría de las veces se trata de una causa funcional, por lo que su tratamiento es sencillo y no requeriría ser derivada a un especialista ${ }^{17}$. En una guía clínica para la evaluación de la constipación en niños elaborada por la Sociedad Norteamericana de Gastroenterología Pediátrica, Hepatología y Nutrición (2006), se recomienda realizar al menos un examen rectal para evaluar la sensación perianal, tono anal y tamaño del recto. Se basan, en que así logran determinar la cantidad y consistencia de las deposiciones y su ubicación dentro del recto ${ }^{18}$. A pesar de estas recomendaciones, el tacto rectal a nivel de atención primaria rara vez es realizado. En un estudio realizado en un centro de referencia de gastroenterología infantil, arrojó que en $77 \%$ de los niños derivados no se había realizado previamente un tacto rectal. De estos niños, $54 \%$ presentaban una impactación fecal. Los autores concluyen que el tacto rectal ayuda a diferenciar las causas orgánicas de las funcionales en la constipación y que podrían alterar el manejo del paciente ${ }^{19}$. Safder y colaboradores también encontraron que la gran mayoría de los niños derivados (85\%) no tuvieron un examen rectal y su omisión produciría derivaciones innecesarias ${ }^{17}$. El tacto rectal es también esencial para diferenciar la encopresis por rebalse de deposiciones, de la encopresis no retentiva ${ }^{20}$. Un estudio cuyo objetivo era determinar la asociación de los hallazgos clínicos con la radiografía simple de abdomen en niños con encopresis, mostró que utilizando la radiografía simple de abdomen como gold standard, el examen rectal tenía un valor predictivo positivo de un $84,8 \%$ y un valor predictivo negativo de un $50 \%$ en la evaluación de retención fecal. Concluyen que un examen rectal positivo es un fuerte predictor de retención fecal, en cuyo caso la radiografía no sería necesaria, aunque un examen rectal negativo puede no descartar totalmente una retención fecal ${ }^{21}$. Sin embargo, una revisión sistemática para evaluar el valor diagnóstico de los hallazgos clínicos en niños con constipación reveló resultados inconsistentes 
con el examen rectal. El "likelihood ratio" de encontrar deposiciones en el examen rectal respecto a la radiografía abdominal es cercano a 1 . De manera que se necesita mayor evidencia que avale el uso de rutina del examen rectal ${ }^{22}$.

\section{Hemorragia gastrointestinal}

Los pólipos colorectales, condición relativamente frecuente en preescolares y escolares, se pueden manifestar a través de hemorragia digestiva baja. Es por ello que se ha recomendado el tacto rectal para identificarlos ${ }^{23}$. En un estudio realizado en niños con pólipos rectales diagnosticados por sigmoidoscopía, $34 \%$ no fueron diagnosticados con el tacto rectal aislado, $25 \%$ tuvieron falsos positivos y un $12 \%$ presentó falsos negativos ${ }^{24}$. En otro estudio realizado en 77 niños con pólipos colorrectales, sólo en 16 de ellos se detectaron los pólipos por medio del tacto rectal $^{25}$. Sin embargo, en la experiencia publicada por Mandhan en el año 2004, en un 64\% se podían palpar los pólipos por medio del tacto rectal ${ }^{26}$.

Otro motivo para realizar tacto rectal, es para la detección de cáncer colorectal. Se recomienda mantener un alto índice de sospecha y realizar un tacto rectal en todo niño que se presente con sangrado ${ }^{27}$. En un reporte de adenocarcinomas rectales en niños, en todos los casos fue posible acceder al tumor a través del tacto rectal. Los autores recomiendan que en todo niño o adolescente que se presente con sangrado rectal, se deba realizar un tacto rectal, además de una proctosigmoidoscopía ${ }^{28}$.

En una revisión reciente en 83 niños con intususcepción, en el 16\% se detectó sangre en el examen rectal con otros hallazgos presentes más comunes, como dolor abdominal, vómitos, palidez, deposiciones sanguinolentas, letargo y masa abdominal. Los autores establecen que es importante considerar otros hallazgos clínicos y no realizar el examen rectal de rutina en caso de sospecha de intususcepción ${ }^{29}$.

\section{Niño con irritabilidad sin causa clara}

En un estudio realizado en niños que consultaban al servicio de urgencia por irritabilidad, sin fiebre, ni otra causa aparente para los padres, la anamnesis reveló la causa en $20 \%$ de los casos, y el examen físico en el $41 \%$ de los casos. Ellos recomiendan realizar un examen físico exhaustivo, incluyendo el examen rectal en forma rutinaria en este escenario ${ }^{30}$. En otro estudio, más reciente, se cuestiona el valor del tacto rectal en niños con irritabilidad sin causa aparente, y afirman que la historia clínica y el examen físico son la piedra angular ${ }^{31}$.

\section{Trauma}

El tacto rectal ha sido considerado como una herramienta esencial para identificar ciertas lesiones ocultas: lesión de intestino bajo, daño de la uretra, fractura de pelvis y lesión de médula espinal. El ATLS (Advanced Trauma Life Support) establece que el tacto rectal es esencial como parte de la evaluación del trauma ${ }^{32}$. El PALS (Advanced Pediatric Life Support), recomienda que el tacto rectal sea realizado por un cirujano experto y sólo si su resultado pudiera alterar el manejo ${ }^{33}$. En un estudio piloto se examinó la utilidad de realizar en forma rutinaria el examen rectal en pacientes pediátricos que habían sufrido un trauma. Se definió como tacto rectal alterado la presencia de sangre abundante $\mathrm{u}$ oculta, la disminución del tono del esfínter rectal, el compromiso de la integridad de las criptas del recto o una próstata alta cabalgante. La sensibilidad y el valor predictivo negativo del examen físico con y sin el tacto rectal eran equivalentes. Los autores concluyen que el tacto rectal de rutina no mejora la identificación de lesiones graves ${ }^{34}$. En otro trabajo similar, que reunió tanto población adulta como pediátrica, los autores concluyeron que omitir este examen parece ser seguro y ventajoso ${ }^{35}$. Shlamovitz y colaboradores, buscaron evaluar las características del tacto rectal en pacientes con trauma y encontraron que la sensibilidad del tacto rectal para detectar cualquier lesión era de un $22,9 \%$, con una especificidad de un 94,7\%. Establecieron que no debe usarse como "screening" en pacientes con trauma ${ }^{36}$.

\section{Otras indicaciones}

Un examen rectal bien realizado tendría importancia ante la sospecha de un teratoma sacrococcígeo, y se recomendaría el examen rectal en todo neonato que se presente con síntomas de obstrucción urinaria baja ${ }^{37}$.

En un estudio prospectivo se evaluó el uso del examen bimanual rectal en casos de testículos no palpables, que posteriormente iban a laparoscopía diagnóstica o a exploración inguinal abierta. La especificidad global fue de un $100 \%$ con una sensibilidad de un $60 \%$. El valor predictivo positivo fue de un $100 \%$. Los autores concluyen que es una herramienta diagnóstica útil. Aunque la sensibilidad no es muy buena, si se lograba palpar, podría evitarse una laparoscopía diagnóstica ${ }^{38}$.

\section{Conclusiones}

Contrario a las enseñanzas tradicionales, numerosos estudios sugieren que someter a los niños a un 
tacto rectal generalmente no aporta más información al diagnóstico y manejo en el caso de sospecha de apendicitis aguda. En los niños que consultan por constipación, el tacto rectal pareciera ser una herramienta útil para diferenciar causas orgánicas de funcionales, y así disminuir las derivaciones innecesarias al policlínico de la especialidad. Sin embargo, la evidencia más reciente vuelve a cuestionar esta práctica. En los niños que consultan por hemorragia gastrointestinal podría recomendarse para la identificación de pólipos o neoplasias. En niños que consultan por irritabilidad, sin una causa aparente, lo más importante es realizar una buena anamnesis y un completo examen físico, y el tacto rectal no debiera ser considerado como parte de una práctica habitual. En el trauma pediátrico el aporte real del tacto real es bajo y otros hallazgos al examen físico pueden sugerir lesiones graves, de manera que puede omitirse. En otras situaciones, como en la sospecha de un teratoma sacrococcígeo y en testículos no palpables, parece ser útil y probablemente se necesiten mayores estudios para recomendar esta práctica.

De esta forma, estimamos que la enseñanza tradicional sobre el uso del tacto rectal en la niñez, debiera ser revisada. En caso de que fuera necesaria su ejecución, debiera ser realizado por un médico experimentado, ya que el procedimiento es incómodo y puede resultar emocional y físicamente traumático, especialmente en las edades pediátricas.

\section{Referencias}

1. Dorland D. Diccionario enciclopédico ilustrado de medicina. 30 Edición Editorial Elsevier 2005; 1871-1872.

2. Hamilton Bailey's demonstrations of physical signs in clinical surgery. Edited by Allan Clain. Fifteenth Edition, Bristol: John Wright \& Sons LTD, 1973.

3. Welch K, Randolph J, Ravitch M, O’Neill J, Rowe M. Pediatric Surgery. Fourth edition, YBMP, 1986, volume II, chapter 88; 105-110.

4. Meneghello J, Fanta E, Paris E, Roselot J. Pediatría. Cuarta edición, Mediterráneo, 1991, volumen I (capítulo 9), volumen II (capítulos 139, 240).

5. Dahlquist LM, Gil KM, Armstrong FD, DeLawyer DD, Greene P, Wuori D. Preparing children for medical examinations: the importance of previous medical experience. Health Psychol 1986; 5: 249-259.

6. Gulla K, Fenheim GE, Myhre AK, Lydersen S. Nonabused preschool children's perception of an anogenital examination. Child Abuse Negl 2007; 31: 885-894.

7. Pate JT, Blount RL, Cohen LL, Smith AJ. Childhood medical experience and temperament as predictors of adult functioning in medical situations. Child Health Care. 1996; 25: 281-298.

8. Osler W. The principles and practices of medicine, designed for the use of practitioners and students of medicine. New York:Appleton, 1901, pág 410.

9. Bryan Ch. Osler: inspirations of a great physician. Published by Oxford University Press, 1997; 164-170.

10. Brewster GS, Herbert ME. Medical myth: a digital rectal examination should be performed on all individuals with possible appendicitis. West J Med 2000; 173: 207 208.

11. Dickson AP, MacKinlay GA. Rectal examination and acute appendicitis. Arch Dis Child 1985; 60: 666-667.

12. Bonello JC, Abrams JS. The significance of a "positive" rectal examination in acute appendicitis. Dis Colon Rectum 1979; 22: 97-101.

13. Bundy DG, Byerley JS, Liles EA, Perrin EM, Katznelson J, Rice HE. Does this child have appendicitis? JAMA 2007; 298: 438-451.

14. Jesudason EC, Walker J. Rectal examination in paediatric surgical practice. Br J Surg. 1999; 86: 376-378. J Pediatr Surg 1986 1; 21: 592-595.

15. Jones PF. Active observation in management of acute abdominal pain in childhood. Br Med J 1976; 2: 551553.

16. Borowitz SM, Cox DJ, Kovatchev B, Ritterband LM, Sheen J, Sutphen J. Treatment of childhood constipation by primary care physicians: efficacy and predictors of outcome.Pediatrics 2005; 115: 873-877.

17. Safder S, Rewalt M, Elitsur Y. Digital rectal examination and the primary care physicians: a lost art? Clin Pediatr (Phila) 2006; 45: 411-414.

18. Constipation Guideline Committee of the North American Society for Pediatric Gastroenterology, Hepatology and Nutrition. Evaluation and treatment of constipation in infants and children: recommendations of the North American Society for Pediatric Gastroenterology, Hepatology and Nutrition. J Pediatr Gastroenterol Nutr 2006; 43: 11-13.

19. Gold DM, Levine J, Weinstein TA, Kessler BH, Pettei MJ. Frequency of digital rectal examination in children with chronic constipation. Arch Pediatr Adolesc Med 1999; 153: 377-379.

20. Schmitt BD. Encopresis. Prim Care 1984; 11: 497-511.

21. Rockney RM, McQuade WH, Days AL. The plain abdominal roentgenogram in the management of encopresis. Arch Pediatr Adolesc Med 1995; 149: 623-627.

22. Reuchlin-Vroklage LM, Bierma-Zeinstra S, Benninga MA, Berger MY. Diagnostic value of abdominal radiography in constipated children: a systematic review. Arch Pediatr Adolesc Med 2005; 159: 671-678.

23. Squires RH Jr. Gastrointestinal bleeding.Pediatr Rev 1999; 20: 95-101.

24. Balkan E, Kiriştioğlu I, Gürpinar A, Ozel I, Sinmaz K, Doğruyol H. Sigmoidoscopy in minor lower gastrointestinal bleeding. Arch Dis Child 1998; 78: 267-268.

25. Pillai RB, Tolia V. Colonic polyps in children: frequently multiple and recurrent. Clin Pediatr (Phila) 1998; 37: 253-257. 
26. Mandhan P. Juvenile colorectal polyps in children: experience in Pakistan.Pediatr Surg Int 2004; 20: 339-342.

27. Pandey A, Gangopadhyay A, Sharma S, Kumar V, Gupta D, Gopal S, et al. Pediatric carcinoma of rectumVaranasi experience. Indian J Cancer 2008; 45: 119-122.

28. Ameh EA, Nmadu PT. Colorectal adenocarcinoma in children and adolescents: a report of 8 patients from Zaria, Nigeria. West Afr J Med 2000; 19: 273-276.

29. Buettcher M, Baer G, Bonhoeffer J, Schaad UB, Heininger $U$. Three-year surveillance of intussusception in children in Switzerland. Pediatrics 2007; 120: 473-480.

30. Poole SR. The infant with acute, unexplained, excessive crying. Pediatrics 1991; 88: 450-445.

31. Freedman SB, Al-Harthy N, Thull-Freedman J. The crying infant: diagnostic testing and frequency of serious underlying disease. Pediatrics 2009; 123: 841-848.

32. American College of Surgeons Committee on Trauma. Advanced Trauma Life Support instructor manual, 6th edn. Chicago, IL: American College of Surgeons; 1997.

33. Advanced Life Support Group. Advanced Paediatric
Life Support: The practical approach. 2nd ed. London: BMJ Publishing Group, 1997.

34. Kristinsson G, Wall SP, Crain EF. The digital rectal examination in pediatric trauma: a pilot study. J Emerg Med 2007; 32: 59-62.

35. Esposito TJ, Ingraham A, Luchette FA, Sears BW, Santaniello JM, Davis KA, et al. Reasons to omit digital rectal exam in trauma patients: no fingers, no rectum, no useful additional information. J Trauma 2005; 59: 1314-1319.

36. Shlamovitz GZ, Mower WR, Bergman J, Crisp J, DeVore HK, Hardy D, et al. Poor test characteristics for the digital rectal examination in trauma patients. Ann Emerg Med 2007; 50: 25-33.

37. Malone PS, Duffy PG, Ransley PG. Urinary tract obstruction secondary to presacral teratoma: a missed diagnosis. J Urol 1989; 142: 1047-1049.

38. Gatti JM, Cooper CS, Kirsch AJ. Bimanual digital rectal examination for the evaluation of the nonpalpable testis. J Urol 2003; 170: 207-210. 